\begin{tabular}{c}
\hline $\begin{array}{c}\text { Brazilian Journal } \\
\text { of Chemical } \\
\text { Engineering }\end{array}$ \\
\hline
\end{tabular}

\title{
DEVELOPMENT AND CHARACTERIZATION OF HIGHLY ORIENTED PAN NANOFIBER
}

\author{
M. Sadrjahani ${ }^{1}$, S. A. Hoseini ${ }^{1}$, V. Mottaghitalab ${ }^{2}$ and A. K. Haghi ${ }^{2 *}$ \\ ${ }^{1}$ Textile Engineering Faculty, Isfahan University of Technology, PO Box 84156, Isfahan, Iran. \\ ${ }^{2}$ Textile Engineering Department, Faculty of Engineering, Phone: +98-131-6690270, \\ Fax: +98-131-6690271, PO Box 3756, University of Guilan, Rasht, Iran. \\ E-mail: Haghi@guilan.ac.ir
}

(Submitted: June 26, 2009 ; Revised: March 13, 2010 ; Accepted: April 23, 2010)

\begin{abstract}
A simple and non-conventional electrospinning technique was employed for producing highly oriented Polyacrylonitrile (PAN) nanofibers. The PAN nanofibers were electrospun from $14 \mathrm{wt} \%$ solution of PAN in dimethylformamid (DMF) at $11 \mathrm{kv}$ on a rotating drum with various linear speeds from $22.5 \mathrm{~m} / \mathrm{min}$ to $67.7 \mathrm{~m} / \mathrm{min}$. The influence of take up velocity was investigated on the degree of alignment, internal structure and mechanical properties of collected PAN nanofibers. Using an image processing technique, the best degree of alignment was obtained for those nanofibers collected at a take up velocity of $59.5 \mathrm{~m} / \mathrm{min}$. Moreover, Raman spectroscopy was used for measuring molecular orientation of PAN nanofibers. Similarly, a maximum chain orientation parameter of 0.25 was determined for nanofibers collected at a take up velocity of $59.5 \mathrm{~m} / \mathrm{min}$.
\end{abstract}

Keywords: Polyacrylonitrile; Nanofiber; Orientation; Eletrospinning.

\section{INTRODUCTION}

With potential applications ranging from protective clothing, tissue engineering and filtration technology to reinforcement of composite nanomaterials, nanofibers offer a remarkable opportunity toward development of multifunctional nanostructural systems (Huang et al., 2003; Ramakrishna et al., 2005; Fennessey et al., 2004; Pan, 2006). The emergence of various applications is inspired by outstanding properties of nanofibers such as huge surface area per mass ratio (Huang et al., 2003) and high porosity along with small pore size (Ramakrishna et al., 2005). Moreover, for diverse applications, highly oriented and flexible nanofiber with superior mechanical properties is extremely demanded. The electrospinning process is a sophisticated technique for producing nanofibers based on applying a high voltage DC electric potential between the end of a capillary tube and a collector. When the applied electric field overcomes the surface tension of the droplet, a charged jet of polymer solution is ejected and nanofibers are collected on the target (Fennessey et al., 2004). Recent studies have shown that aligned nanofibers have better molecular orientation and, as a consequence, improved mechanical properties than randomly oriented nanofibers (Fennessey et al., 2004; Zussman et al., 2005; Gu et al., 2005). Additionally, the aligned nanofibers are better suited for preparing carbon nanofibers from electrospun PAN nanofiber precursors (Jalili et al., 2006). In another attempt (Fennessey et al., 2004) tows of unidirectional and molecularly oriented PAN nanofibers were prepared using a high speed, rotating take up wheel. A maximum orientation factor of 0.23 was determined for nanofibers collected between $8.1 \mathrm{~m} / \mathrm{s}$ and $9.8 \mathrm{~m} / \mathrm{s}$. The aligned tows were twisted into yarns, and the mechanical properties of the yarns were determined as a function of twist angle. Their produced yarn with twist angle of $11^{\circ}$ had an initial modulus and ultimate strength of about $5.8 \mathrm{GPa}$ and $163 \mathrm{MPa}$, respectively (Fennessey et al., 2004). Zussman et al. (2005) have

*To whom correspondence should be addressed 
demonstrated the use of a wheel-like bobbin as the collector to position and align individual PAN nanofibers into parallel arrays with an orientation factor of 0.34 at a collection speed of $5 \mathrm{~m} / \mathrm{s}$. In another study, aligned PAN nanofibers collected across the gap between the two grounded strips of aluminum foil showed an obvious improvement of mechanical properties in the modulus of the resultant carbon nanofibers, as a consequence of an increase in the orientation factor from 0 to 0.127 (Gu et al., 2005).

The current study focuses on preparation of aligned and molecularly oriented PAN nanofibers using two needle pumps in a highly productive approach. Various take up velocities were examined to obtain the highest possible collection speed capable of producing aligned nanofiber. Nondestructive techniques like angular power spectra (APS) and Raman spectroscopy were utilized for characterization of alignment and molecular orientation in nanofibers.

\section{EXPERIMENTAL}

\section{Material and Reagents}

Industrial polyacrylonitrile (PAN) with average molecular weight of $100000 \mathrm{~g} / \mathrm{mol}$ and highly pure dimethylformamide (DMF, 99\%) were supplied respectively by Iran Polyacryle and Merck companies. All PAN/DMF solutions were prepared using constant power magnetic stirrer at room temperature.

\section{Electrospinning Setup}

The electrospinning apparatus consists of a high voltage power supply; two syringe pumps, two stainless steel needles $(0.7 \mathrm{~mm}$ OD) and a rotating collector equipped to an inverter for controlling linear speed (Fig 1). In this setup, unlike the conventional technique, two needles were installed in opposite directions and polymer solutions were pumped to needles by two syringe infusion pumps with same injection rate. The flow rate of solutions to the needle tip is maintained constant so that a pendant drop remains during electrospinning. The horizontal distance between the needles and the collector was $20-25 \mathrm{~cm}$. When high voltages (9-11 $\mathrm{KV}$ ) were applied to the needles with opposite voltage, jets were ejected simultaneously. Then the jets with opposite charges attracted each other, stuck together and a cluster of fibers is formed. For collecting aligned nanofibers, the cluster of fibers formed between the two needles was towed manually to a rotating drum.

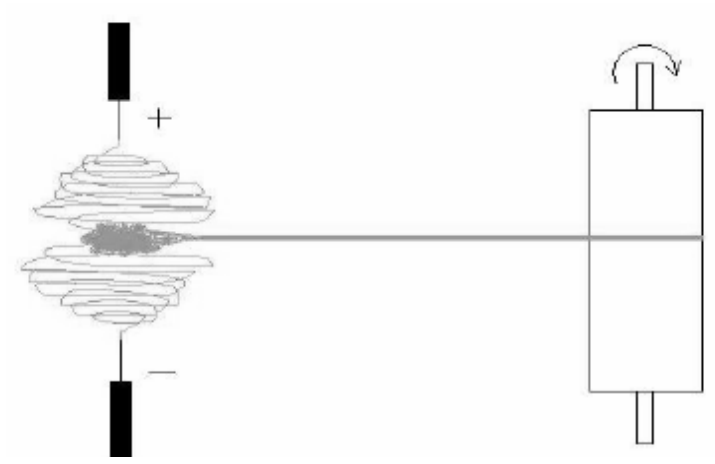

Figure 1: Schematic of electrospinning setup for collection of continuous aligned nanofiber

\section{Characterization Techniques}

Various characterization techniques were applied to 10 nanofiber samples produced in similar conditions. The geometry of electrospun nanofibers was investigated using a Philips XL-30 scanning electron microscope (SEM). Nanofiber samples were mounted onto SEM plates; sputter coated with gold and inserted in a SEM vacuum vessel for image capturing. Also, the Motic optical microscope was employed to acquire images for alignment analysis. Fiber alignment was characterized using image-processing technique by Fourier Power Spectrum (FPS) as a function of take up speed. All images used in the process were obtained at a resolution of $640 \times 480$ pixels and 1000 times magnifications. The number of captured images was 30 at each of the take up speeds. The variation of molecular orientation with respect to take up speed was characterized by Polarized Raman spectroscopy. Raman spectra were obtained with a Thermo Nicolt Raman Spectrometer model Almega Dispersive 5555. The spectra were collected in the backscattering mode, using the $532 \mathrm{~nm}$ line of a Helium/Neon laser. The nominal power of the laser was $30 \mathrm{~mW}$. The nanofiber axis is oriented at an angle of $\theta^{\circ}$ with respect to the machine direction. The angle between the polarization plane and the nanofiber axis is $\psi$. The orientation studies were performed when fibers were at $\psi=0^{\circ}$ and $\psi=90^{\circ}$ to the plane of polarization of the incident laser. At each angle, the enhanced spectra at VV (polarized laser and the analyzer are parallel) and $\mathrm{VH}$ (the polarized laser and the analyzer are perpendicular) configurations were collected. 


\section{RESULTS AND DISCUSSION}

Well-aligned PAN nanofibers with very long lengths were electrospun using a simple and unconventional method. In this technique, the electric field only exists between two syringe needles (not between needle ad collector) and, thus, the movement of nanofibers in the drawing area (distance between the needles and the collector) is due to mechanical force caused by the rotating drum and electric field and therefore has no impact on the drawing mechanism. Various operational and material parameters were adjusted in order to obtain optimum and productive conditions. PAN nanofibers prepared at 14 and 15 $\mathrm{wt} \%$ have lower rupture compared to $13 \mathrm{wt} \%$ polymer solution, presumably due to a higher level of chain entanglement. The conditions pertinent to minimum number of rupture for nanofibers prepared at different concentrations are shown in Table 1.

Fig 2 shows SEM images of PAN nanofibers electrospun in optimum conditions. The generated nanofibers have uniform structure without any bead formation. The average diameter of nanofibers increases with increasing polymer concentration because of lower extension of the jet at higher concentration and viscosity. Based on SEM images, the average diameter of nanofibers produced from 13 $\mathrm{wt} \%$ polymer solutions is about $323 \pm 31 \mathrm{~nm}$, which is about $100 \mathrm{~nm}$ less than the diameter of those fibers collected from $15 \mathrm{wt} \%$ polymer solution (Ziabari et al., 2009). This is probably due to the greater stretching resistance of the solution with increasing solid content (Ramakrishna et al., 2005). However the $14 \mathrm{wt} \%$ concentration was chosen for producing of PAN nanofibers due to a lower number of rupture and higher diameter uniformity.

Alignment analysis of nanofibers collected at different take up speeds from $22.5 \mathrm{~m} / \mathrm{min}$ to 67.7 $\mathrm{m} / \mathrm{min}$ was carried out using the angular power spectrum (APS) based on micrographs captured by an optical microscope. Acquired image was processed using MATLAB software to produce angular power spectrum as the square of the Fourier power spectrum. APS is a plot of how much the orientation varies from point to point on the web of nanofibers versus the angular frequency (the $\mathrm{x}$-axis variable). The sharpness of the peak at an angle of $90^{\circ}$ on the APS shows the degree of alignment of nanofibers in the vertical direction and the area of that peak shows the density of aligned nanofibers (Jalili et al., 2006). Furthermore, the plot of the normalized APS (ratio of intensity of the APS to the corresponding mean intensity of the Fourier power spectrum) versus angle was used for calculating the degree of alignment (Fig 3). Disorientation of nanofibers generates a broad spectrums around $90^{\circ}$, which reflects directly a low degree of alignment. The ratio of peak area at $90^{\circ} \pm 3^{\circ}$ to total area of the APS plot was utilized to calculate the density of aligned nanofibers. Table 2 shows the influence of the linear take up speed of the rotating collector on the degree of alignment of the nanofibers.

Table 1: Various conditions used for nanofiber preparation

\begin{tabular}{|c|c|c|c|c|c|}
\hline $\begin{array}{c}\text { Solution concentration } \\
(\mathbf{w t} \%)\end{array}$ & $\begin{array}{c}\text { Applied voltage } \\
\mathbf{( k v )}\end{array}$ & $\begin{array}{c}\text { Feed rate } \\
(\mathbf{m L} / \mathbf{h})\end{array}$ & $\begin{array}{c}\text { Distance between } \\
\text { two needles } \\
(\mathbf{c m})\end{array}$ & $\begin{array}{c}\text { Distance between } \\
\text { needles and collector } \\
\text { (cm) }\end{array}$ & $\begin{array}{c}\text { Total number of } \\
\text { rupture at } \\
(\mathbf{1 5} \text { minutes) }\end{array}$ \\
\hline 13 & 10.5 & 0.293 & 13 & 20 & 12 \\
1 & 11 & 0.293 & 13 & 20 & 6 \\
15 & 11 & 0.293 & 15 & 20 & 6 \\
\hline
\end{tabular}

${ }^{1}$ It was measured visually by counting the number of rupture of fiber flow between electrospinning needles and rotating collector.

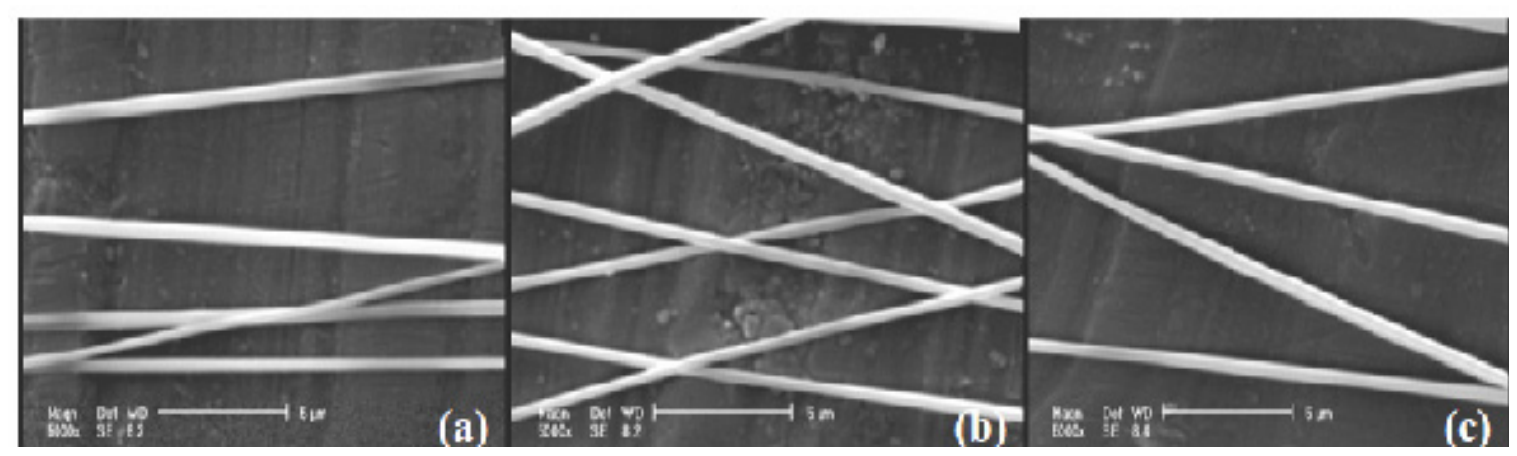

Figure 2: SEM images of PAN nanofibers (a) $13 \mathrm{wt} \%$ (b) $14 \mathrm{wt} \%$ (c) $15 \mathrm{wt} \%$ polymer solution 

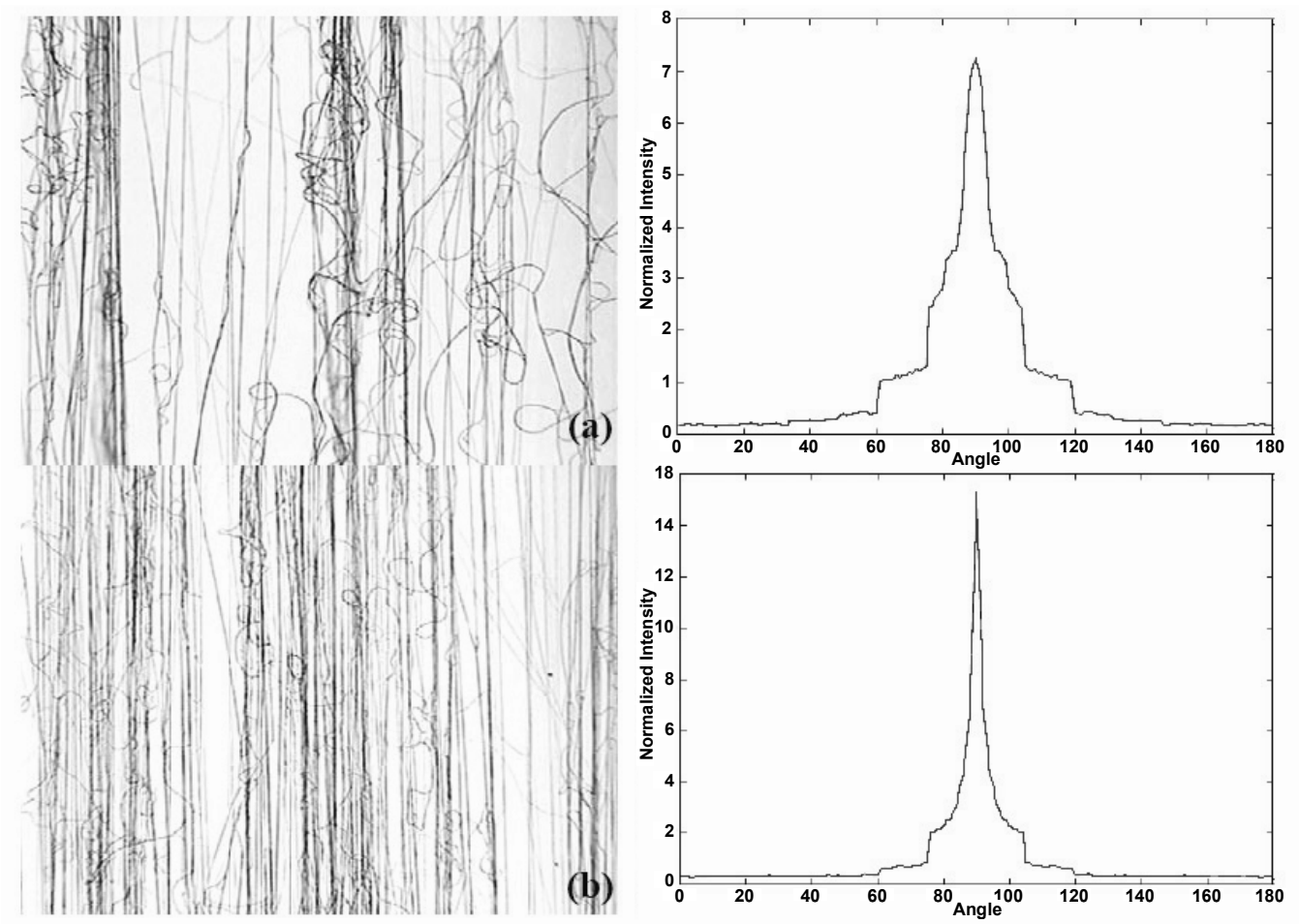

Figure 3: Optical micrograph of electrospun PAN nanofibers with the corresponding normalized APS at take up speeds of (a) $22.5 \mathrm{~m} / \mathrm{min}$ (b) $59.5 \mathrm{~m} / \mathrm{min}$

Table 2: The degree of alignment at different take-up speeds

\begin{tabular}{|l|c|c|c|c|c|c|}
\hline Take up speed & 22.5 & 31.6 & 40.6 & 49.6 & 59.5 & 67.7 \\
\hline Degree of alignment & $24.6 \pm 3.9$ & $34.4 \pm 5.2$ & $32.7 \pm 7.65$ & $29.5 \pm 5.97$ & $37.5 \pm 5.2$ & $29.4 \pm 7.1$ \\
\hline
\end{tabular}

The higher the take up speed, the more aligned is the nanofiber collected. As the rotation speed increases, the effective draw (difference between the surface velocity of the drum and the final velocity of the fiber) is increased, resulting in better alignment of the collected fiber and less deviation between the fiber and rotation direction. However, the positive impact of linear take up velocity on degree of alignment passes through a maximum of $37.5 \%$ at $59.5 \mathrm{~m} / \mathrm{min}$ and the trend of the data reverses after this collection speed. The decrease of the degree of alignment after a specific take up speed presumably is due to insufficient time for arrangement of the molecular chains in the drawing mechanism. The drawing mechanism inherently uncoils the molecular chain to reach higher orientation, but after an optimum take up speed the breaking force acting on the molecular chain starts to build up. At the early stage, the decrease of nanofiber alignment can be observed due to partial segregation of the polymer chains and reforming of the coiled structure. Meanwhile, if the take up speed increase is continued, complete fracture occurs in the nanofiber structure. The molecular orientation of the nanofibers was also characterized using Polarized Raman Spectroscopy. Figure 4 shows the Raman spectra of nanofiber samples collected at different take up speeds under the VV configuration at $\psi=0^{\circ}$ (parallel) and $90^{\circ}$ (Vertical) versus the polarization plane. Four samples with symbols A, B, C and D were prepared using conditions given in Table 1 for $14 \mathrm{wt} \%$ polymer solution and various rotating drum speeds. Raman spectra of PAN nanofibers usually arise in the region of $500-1500 \mathrm{~cm}^{-1}$ which is commonly observed as the Raman fingerprint of PAN microfiber (Huang and Koenig 1971). Compared to the peak enhancement at $600 \mathrm{~cm}^{-1}$ with constant intensity at different polarization angles, the intensity at $1394 \mathrm{~cm}^{-1}$ monotonically decreases with increasing $\psi$. The intensity dependence of the peak enhancement at $1394 \mathrm{~cm}^{-1}$ on the angle between fiber and polarization plane can be considered as a powerful tool for determination of nanofiber orientation. Other peaks that enhanced between 1394 $\mathrm{cm}^{-1}$ and $600 \mathrm{~cm}^{-1}$ did not decrease significantly, except the peak enhanced at $1190 \mathrm{~cm}^{-1}$. 


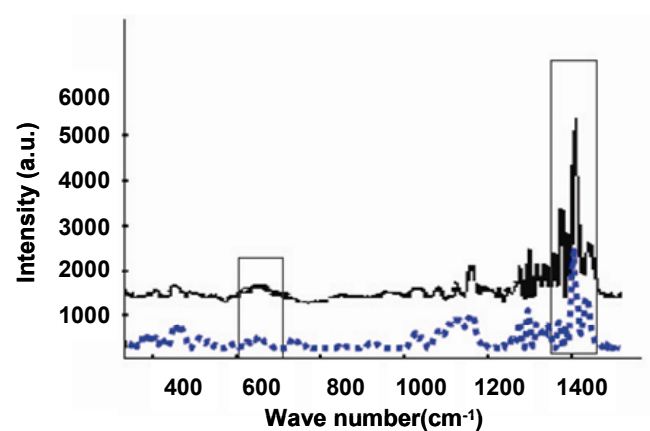

(a)

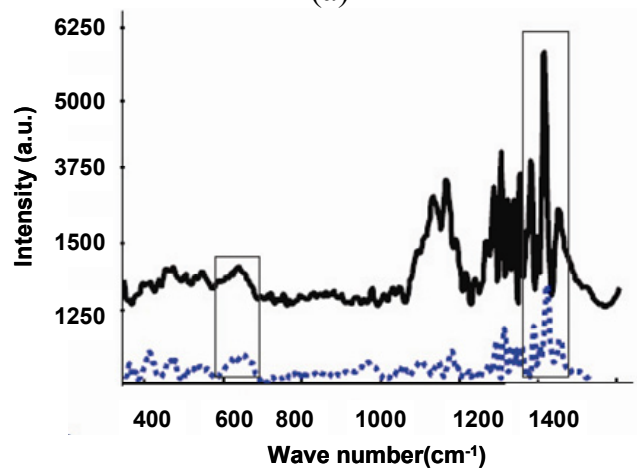

(c)

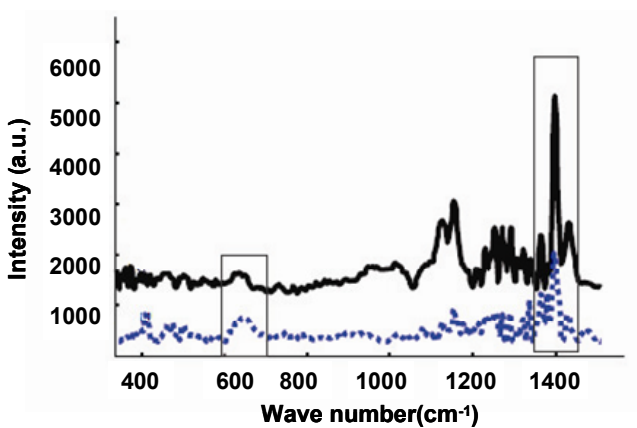

(b)

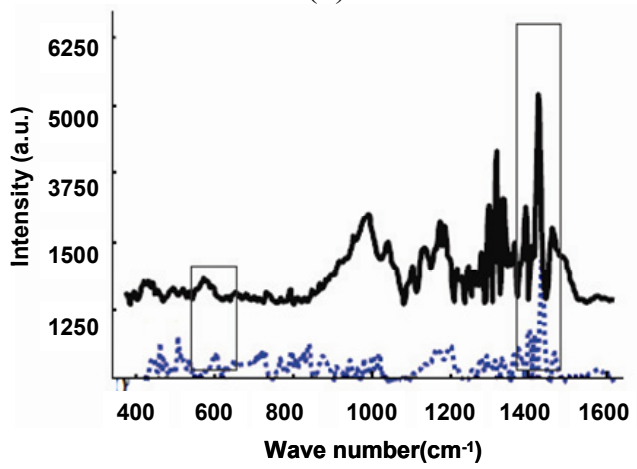

(d)

Figure 4: The Raman spectra under the VV mode: (a) $22.5 \mathrm{~m} / \mathrm{min}$; (b) $49.6 \mathrm{~m} / \mathrm{min}$; (c) $59.5 \mathrm{~m} / \mathrm{min}$; (d) $67.7 \mathrm{~m} / \mathrm{min}$. The angle between the fiber axis and the polarization plane for filled curve and dashed curve are $0^{\circ}$ and $90^{\circ}$, respectively.

Similar to the VV configuration, the peak intensity observed for different samples in the $\mathrm{VH}$ configuration at $\psi=0$ characterized the degree of molecular orientation. According to the intensity ratios shown in Table 3, Raman spectra show a much stronger orientation effect in sample (C) compared to other spun nanofibers. It is clear that the higher take up speed is mostly responsible for the orientation of the molecular chains in the fiber direction. Therefore, as expected, a low orientation dependence of the Raman modes was observed for the lower take up speed. The orientation parameters of $\langle\mathrm{P} 2(\cos \theta)>$ and $<\mathrm{P} 4(\cos \theta)>$, which are, respectively, the average values of the Legendre polynomials $\mathrm{P} 2(\cos \theta)$ and $\mathrm{P} 4$ $(\cos \theta)$ for the bulk product, can be calculated based on the Raman intensity ratios in $\mathrm{VV}$ and $\mathrm{VH}$ modes given in Table 3 (Liu and Kumar 2003).

More specifically, $\langle\mathrm{P} 2(\cos \theta)>$ is known as the Herman orientation factor (f), which varies between values of 1 and 0 , corresponding, respectively, to a nanofiber fully oriented in the take up direction and a fully non-oriented nanofiber distribution. The Herman orientation factor for different samples varies between 0.20 and 0.18 when the take up speed increases from $22.5 \mathrm{~m} / \mathrm{min}$ to $67.7 \mathrm{~m} / \mathrm{min}$ (Fig 5-a).

It can be clearly seen that the degree of orientation reaches a maximum point at $59.5 \mathrm{~m} / \mathrm{min}$ and then decreases at higher take-up speed. This trend is in agreement with data acquired by optical microscopy through the normalized APS, as shown in previous work (Fennessey et al., 2004). The orientation parameter decreases for greater take up speeds probably due to the decrease of the time necessary for drawing nanofibers; as a result, there is a lack of sufficient opportunity for arranging the molecular chains in the tension direction. Comparing the higher molecular orientation parameter (up to 0.25 at a take up speed of $59.5 \mathrm{~m} / \mathrm{min} \sim 1 \mathrm{~m} / \mathrm{s}$ ) achieved in the current study with that of other methods shows that this technique had a more effective influence on the molecular orientation of nanofibers than other methods (Fennessey et al., 2004; Zussman et al., 2005). In other cases in which two grounded strips were used as a collector, the obtained value of the chain orientation parameter (0.127) was less than in our study (Zussman et al., 2005). On the other hand, using the rotating drum as a collector led to the measured orientation parameter of 0.23 at higher take up speed $(9.84 \mathrm{~m} / \mathrm{s})$ (Fennessey et al., 2004). Therefore it can be speculated that, considering the more effective applied stretch in this system, the presented above method will help to reach suitable molecular orientations at lower take up speeds. 
Table 3: The intensity ratios of the enhanced peak at $1394 \mathrm{~cm}^{-1}$ using $\mathrm{VV}$ and VH configuration

\begin{tabular}{|c|c|c|c|c|c|c|c|}
\hline Sample & $\begin{array}{c}\text { Rotating drum } \\
\text { Speed (m/min) }\end{array}$ & $\mathbf{I}_{\mathbf{V V 0}}$ & $\mathbf{I}_{\mathbf{V V 9 0}}$ & $\mathbf{I}_{\mathbf{V H} \mathbf{0}}$ & $\mathbf{I}_{\mathbf{V V 9 0} \mathbf{0}} / \mathbf{I}_{\mathbf{V H} \mathbf{0}}$ & $\mathbf{I}_{\mathbf{V V 0}} / \mathbf{I}_{\mathbf{V H} \mathbf{0}}$ & $\mathbf{I}_{\mathbf{V V 0}} / \mathbf{I}_{\mathbf{V V 9 0}}$ \\
\hline $\mathrm{A}$ & 22.5 & 4400 & 1900 & 1600 & 1.19 & 2.75 & 2.31 \\
$\mathrm{~B}$ & 49.6 & 3600 & 1500 & 2400 & 0.63 & 1.50 & 2.40 \\
$\mathrm{C}$ & 59.5 & 4000 & 1500 & 4277 & 0.35 & 0.93 & 2.67 \\
$\mathrm{D}$ & 67.7 & 3800 & 2200 & 6350 & 0.35 & 0.60 & 1.73 \\
\hline
\end{tabular}

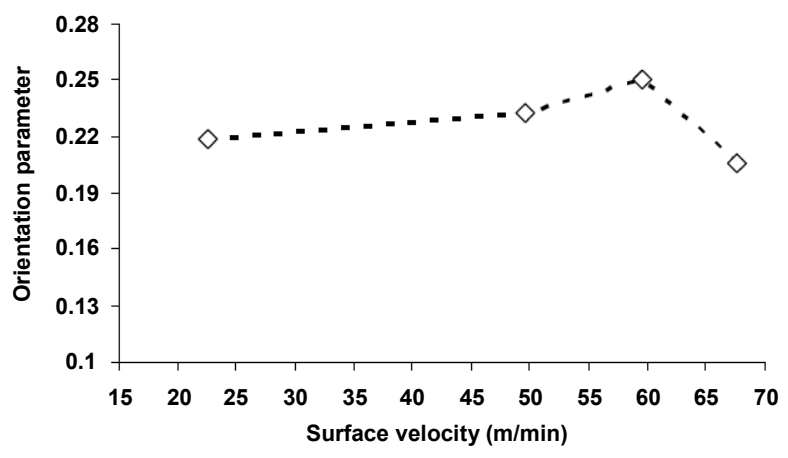

(a)

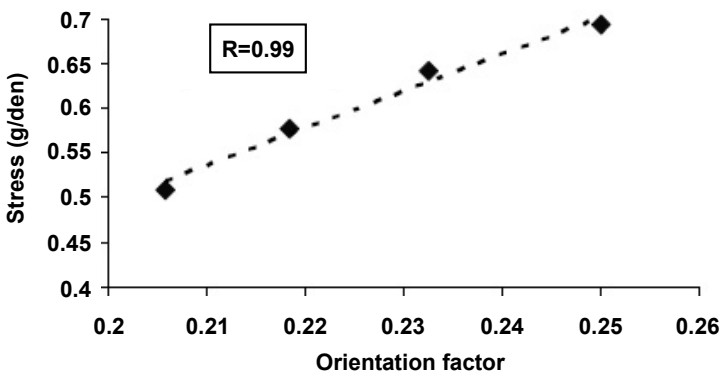

(b)

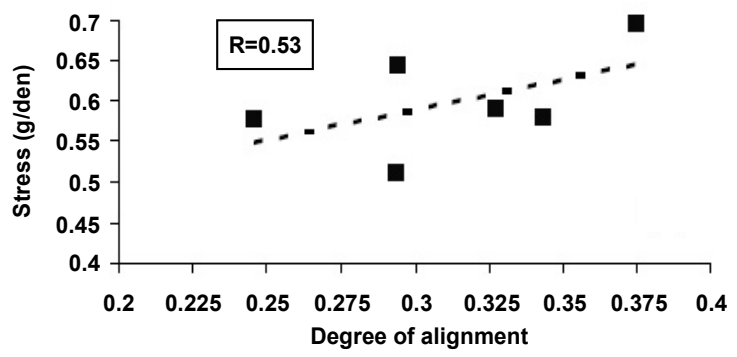

(c)

Figure 5: (a) Orientation parameter versus take up speed determined by Raman spectroscopy; (b) Stress versus molecular orientation parameter; (c) Stress versus degree of alignment.

Figures 5 (b), (c) represent, respectively, the plots of stress versus molecular orientation parameter and degree of alignment. The higher the degree of alignment, the greater the number of fibers oriented in the tension direction. The higher correlation factor of 0.99 (stress- orientation factor curve) compared to 0.53 (stress- degree of alignment curve) suggests that the molecular orientation is more responsible for good mechanical properties of the resultant nanofibers than degree of alignment. The former factor is at the molecular level and controls the degree of crystallinity and, therefore, the macroscopic properties.

\section{CONCLUSION}

A simple and non-conventional electrospinning technique using two syringe pumps was employed for producing highly oriented polyacrylonitrile
(PAN) multifilament nanofibers. The process was carried out using two needles in opposite positions and a rotating collector perpendicular to the needle axis. The current procedure was optimized for increasing of orientation and productivity of nanofibers with diameters in the nanoscale range. PAN nanofibers were electrospun from $14 \mathrm{wt} \%$ solutions of PAN in dimethylformamide (DMF) at $11 \mathrm{kv}$ on a rotating drum with various linear speeds from $22.5 \mathrm{~m} / \mathrm{min}$ to $67.7 \mathrm{~m} / \mathrm{min}$. The influence of take up velocity on the degree of alignment, internal structure and mechanical properties of collected PAN nanofibers was also investigated. Various characterization techniques were employed to find out the influence of operational parameters on the degree of orientation. Based on micrographs captured by optical microscopy, the angular power spectrum (APS) was generated based on an image processing technique. The best degree of alignment 
was obtained for those nanofibers collected at a take up velocity of $59.5 \mathrm{~m} / \mathrm{min}$. Moreover, polarized Raman spectroscopy under $\mathrm{VV}$ configuration at $\psi=$ $0^{\circ}$ (parallel) and $90^{\circ}$ (Vertical) versus polarization plane and also in $\mathrm{VH}$ configuration at $\psi=0$ was used as a standard technique for measuring molecular orientation of PAN nanofibers. Similarly, a maximum chain orientation parameter of 0.25 was determined for nanofibers collected at a take up velocity of $59.5 \mathrm{~m} / \mathrm{min}$.

\section{REFERENCES}

Fennessey, S. F. and Farris, R. J., Fabrication of aligned and molecularly oriented electrospun polyacrylonitrile nanofibers and the mechanical behavior of their twisted yarns. Polymer, 45, 4217 (2004).

Gu, S. Y., Ren, J. and Wu, Q. L., Preparation and structures of electrospun PAN nanofibers as a precursor of carbon nanofibers. Synthetic Metals, 155, 157 (2005).

Huang, Y. S. and Koenig, J. L., Raman spectra of polyacrylonitrile. Applied Spectroscopy, 25, 620622 (1971).

Huang, Z. M., Zhang, Y. Z., Kotaki, M. and Ramakrishna, S., A review on polymer nanofibers by electrospinning and their applications in nanocomposites. Composites Science and Technology, 63, 2223 (2003).

Jalili, R., Morshed, M. and Hosseini Ravandi, S. A., Fundamental Parameters Affecting Electrospinning of PAN Nanofibers as Uniaxially Aligned Fibers. Journal of Applied Polymer Science, 101, 4350 (2006).

Liu, T. and Kumar, S., Quantitative characterization of SWNT orientation by polarized Raman spectroscopy. Chem. Phys. Lett, 378, 257 (2003).

Pan, H., Li, L., Hu, L. and Cui, X., Continuous aligned polymer fibers produced by a modified electrospinning method. Polymer, 47, 4901 (2006).

Ramakrishna, S., Fujihara, K., Teo, W. E., Lim, T. C. and Ma, Z., An introduction to electrospinning and nanofibers. World Scientific, Singapore (2005).

Ziabari, M., Mottaghitalab, V. and Haghi, A. K. Application of direct tracking method for measuring electrospun nanofiber diameter. Braz. J. Chem. Eng., 26, 1, 53 (2009).

Zussman, E., Chen, X., Ding, W., Calabri, L., Dikin, D. A., Quintana, J. P. and Ruoff, R. S., Mechanical and structural characterization of electrospun PAN-derived carbon nanofibers. Carbon, 43, 2175 (2005). 